Original Paper http://ajol.info/index.php/ijbcs http://indexmedicus.afro.who.int

\title{
Etude ethnobotanique des Asteraceae médicinales vendues sur les marches du district autonome d'Abidjan (Côte d'Ivoire)
}

\author{
Adon Basile YAPI ${ }^{{ }^{*}}$, N'Dja Justin KASSI ${ }^{1}$, N'Guessan Bra Yvette FOFIE ${ }^{2}$ et \\ Guédé Noël ZIRIHI ${ }^{1}$

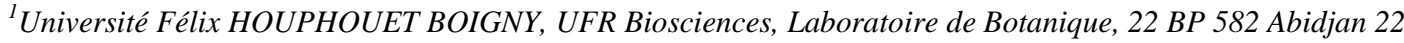 \\ (Côte d'Ivoire). \\ ${ }^{2}$ Université Félix HOUPHOUET BOIGNY, UFR Sciences Pharmaceutiques et Biologiques, Laboratoire de \\ Pharmacognosie, Botanique et Cryptogamie, 22 BP 582 Abidjan 22 (Côte d'Ivoire). \\ *Auteur correspondant, E-mail : adonbasile@yahoo.fr ou kassindja@yahoo.fr
}

\section{RESUME}

L'utilisation des plantes de notre environnement immédiat dans les soins de santé primaire en Afrique et surtout chez les populations pauvres, constitue une pratique très courante. Une enquête ethnobotanique menée auprès de 110 herboristes des marchés du district autonome d'Abidjan (Côte d'Ivoire) a permis de répertorier 27 espèces végétales appartenant à la famille des Asteraceae. Ces espèces sont regroupées en 20 genres et 7 tribus. Le genre Vernonia $(22,22 \%)$ est le plus représenté. Les spectres morphologie et biologique montrent une prédominance d'herbes $(85,19 \%)$ et de thérophytes $(44,45 \%)$. Ces Asteraceae sont utilisées dans la formulation de 57 recettes pour combattre 70 affections. Les feuilles $(43,18 \%)$ sont les organes les plus prisés. Le pétrissage $(38,60 \%)$ et la décoction $(33,34 \%)$ sont les techniques de préparation médicamenteuse les plus utilisées. Le décocté, le jus et la pâte sont les formes médicamenteuses couramment prescrites et fréquemment administrées en boisson $(35,94 \%)$. Les maladies les plus fréquemment cités sont le paludisme, les maladies infantiles et la grossesse. Les Asteraceae les plus sollicitées sont Acanthospermum hispidum, Ageratum conyzoides, Vernonia amygdalina. Il ressort de diverses études phytochimiques que l'effet curatif des plantes médicinales est le fait de métabolites primaires et surtout secondaires.

(C) 2015 International Formulae Group. All rights reserved.

Mots clés : Asteraceae, ethnobotanique, herboristes, plantes médicinales, Abidjan, Côte d'Ivoire.

\section{Ethnobotanic study of medicinal Asteraceae sold on the markets of the autonomous district of Abidjan (Côte d'Ivoire)}

\begin{abstract}
The use of plants in our immediate environment in primary health care in Africa and especially in the poor populations is a very common practice. An ethnobotanical survey, carried out on 110 herbalists of the
\end{abstract}


markets of the autonomous district of Abidjan (Côte d'Ivoire), has enabled to identify 27 plant species belonging to the family of Asteraceae. These species are grouped into 20 genera and 7 tribes. The genus Vernonia (22.22\%) is the most represented. The morphological and biological analyses show a predominance of herbs $(85.19 \%)$ and therophytes $(44.45 \%)$. These Asteraceae are used in the formulation of 57 recipes to fight against 70 diseases. The leaves $(43.18 \%)$ are the most valuable organs. Kneading (38.60\%) and the decoction (33.34\%) are drug preparation techniques most used. The decocted, the juice and the paste are the pharmaceutical forms commonly prescribed and frequently administered as drink (35.94\%). The most frequently cited diseases are malaria, infantile diseases and pregnancy. The most requested of the Asteraceae are Acanthospermum hispidum, Ageratum conyzoides, Vernonia amygdalina. It is observed from the various phytochemical studies that the curative effect of the medicinal plants is the fact of primary and especially secondary metabolites.

(C) 2015 International Formulae Group. All rights reserved.

Keywords: Asteraceae, ethnobotanic, herbalists, medicinal plants, Abidjan, Côte d'Ivoire.

\section{INTRODUCTION}

Dans les pays en développement, notamment en Afrique, malgré les progrès de la médecine moderne, certaines affections chroniques non transmissibles (maladies cardiovasculaires, diabète, cancer...) et transmissibles (SIDA, hépatites virales, paludisme...), demeurent le plus souvent invincibles et sont sources de nombreux cas de décès. Selon Hossain et al. (2007), les maladies cardiovasculaires sont responsables d'environ 18 millions de décès dans le monde et d'ici 2030 , le nombre est estimé à près de 23,6 millions. L'émergence de ces maladies dans nos sociétés est due à un contexte socioéconomique peu propice, une insuffisance et une mauvaise réputation du personnel de santé. Mais, la raison la plus importante est le coût élevé de certains médicaments pharmaceutiques (Gning et al., 2007). Aujourd'hui, le paludisme est une maladie considérée comme une cause de pauvreté (OMS, 2004). Que faire? Pour apporter un élément de réponse à cette question, il serait nécessaire de rechercher, parmi les ressources naturelles, des thérapeutiques nouvelles, d'accès facile, peu onéreuses, susceptibles de traiter efficacement diverses affections. C'est ainsi que de nombreuses investigations ethnopharmacologiques ont été menées en
Afrique et dans la plupart des pays en développement (Eddouks et al., 2007; Sangaré et al., 2012; Gueye et al., 2012) notamment en Côte d'Ivoire (Tra Bi et al., 2008; Azokou, 2010, Lebri et al., 2015). Les enquêtes ethnopharmacologiques menées le plus souvent, visent une affection définie entraînant le recensement des plantes appartenant à diverses familles botaniques. Cependant, celles visant les espèces végétales d'une famille botanique spécifique restent insuffisantes pour la pharmacopée ivoirienne. C'est ainsi que cette étude qui est un travail préliminaire, sur la recherche dans l'environnement immédiat des populations, des ressources végétales, utilisées dans le traitement de diverses affections. Les objectifs de ce travail consistent à répertorier, documenter et cataloguer les savoirs traditionnels sur les Asteraceae médicinales commercialisées. Préciser en accord avec la littérature, les bases scientifiques qui permettent d'expliquer l'utilisation thérapeutique traditionnelle des plantes.

\section{MATERIEL ET METHODES}

\section{Présentation de la zone d'étude}

La zone d'étude est le District Autonome d'Abidjan, situé au Sud de la Côte d'Ivoire. Avec une superficie de $2119 \mathrm{~km}^{2}$, le 
district est limité au Nord par les départements de Sikensi et d'Agboville, au Sud par l'océan Atlantique, à l'Ouest par les départements de Dabou et de Jacqueville et à l'Est par les départements d'Alépé et de Grand-Bassam. Ses coordonnées géographiques sont $5^{\circ} 20^{\prime} 11^{\prime \prime}$ de latitude Nord et entre $4^{\circ} 01^{\prime} 36^{\prime \prime}$ de longitude Ouest. En plus des 3 souspréfectures, l'étude a été également réalisée dans 8 communes du district dont 4 (Adjamé, Abobo, Yopougon et Port-Bouët) constituent pour les marchés, des portes d'entrées Ouest, Nord et Est d'Abidjan, en plantes médicinales. La commune d'Adjamé au centre du district possède l'un des plus grands marchés de vente de gros des plantes médicinales.

\section{Matériel végétal et technique}

Le matériel végétal est représenté par l'ensemble des plantes faisant l'objet de cette étude. Comme matériel technique, un matériel classique permettant de mettre des échantillons en herbier a été utilisé. Un appareil à photographie numérique de type Nikon a permis les prises de vue et une balance électronique pour les pesées.

\section{Collecte des données}

Une étude préliminaire de reconnaissance des espèces végétales de la famille des Asteraceae recensées en Côte d'Ivoire (Aké-Assi, 2001), est menée. Pour cette étude, des flores, des ouvrages, l'outil internet et des spécimens de l'herbier du Centre National Floristique (CNF) d'Abidjan, sont consultés. Une espèce est retenue lorsqu'elle est relativement commune et que sa répartition est large et également si son utilisation traditionnelle est courante.

La méthode d'échantillonnage stratifié probabiliste (Tra Bi et al., 2008) est utilisée. Elle consiste à diviser la zone d'étude en différentes strates et à y associer le même nombre d'enquêtés. Dans ce travail, chaque commune a constitué une strate. Dans chacune des strates, différents marchés ont été visités, et 10 commerçants de plantes médicinales sont interviewés. Ces interviews sont assemblées pour constituer un échantillon global de 110 herboristes du district autonome d'Abidjan. Des enquêtes ethnobotaniques basées sur la méthode d'entretien semistructuré (Klotoé et al., 2013) sont conduites dans 33 marchés de ces 11 communes. Les critères de choix des herboristes ont résidé dans la richesse et le volume de plantes de leur étalage ainsi que de la présence des espèces végétales de la famille des Asteraceae. Chaque entretien était accompagné de l'achat de drogues, pour connaitre la partie de la plante utilisée et leur prix afin de pouvoir évaluer leur valeur marchande et aussi en échange des informations relatives aux vertus thérapeutiques de chaque espèce. Ces achats ont constitué des récoltes de spécimens qui sont identifiés par comparaison à l'herbier du CNF, de l'Université Félix HOUPHOUETBOIGNY. Pour nommer les plantes recensées, la classification phylogénétique, selon les travaux de l'Angiosperms Phylogeny Group, dans sa dernière version dite APG III, est adoptée. En ce qui concerne les ordres, les clades, les sous-phylums et les phylums, le même principe est suivi. Les termes utilisés pour désigner les types biologiques et les types phytogéographiques sont empruntés à Aké-Assi (2001). Des informations sur les enquêtés et les Asteraceae médicinales proposées, sont collectées. Les indications sur le nombre de bottes de chaque drogue vendue ont été nécessaires pour établir le taux de vente. Des données phytochimiques de la littérature ont permis de comprendre et de justifier les utilisations thérapeutiques traditionnelles des plantes.

\section{Traitement des données}

A partir des variables échantillonnées, notamment le profil de chaque enquêté (sexe, 
âge, niveau d'études, ethnie), la caractérisation des commerçants de notre zone d'étude, a été possible. Pour chaque plante, les données collectées sont composées du nom vernaculaire, du nom scientifique, de l'organe utilisé, du mode de préparation et d'administration ainsi que du prix de vente. La méthode d'analyse a consisté à déterminer les paramètres que sont la fréquence d'utilisation des Asteraceae pour traiter les affections et le taux de vente en vue de faire une analyse statistique de ces données. A ces paramètres, s'est ajoutée la valeur marchande qui a permis d'apprécier la rentabilité financière par la vente des plantes médicinales. Les données sont enregistrées sur un tableur Excel, puis analysées avec le logiciel Excel 2010.

\section{RESULTATS}

Répertoire des espèces végétales médicinales de la famille des Asteraceae répertoriés

Les investigations ethnobotaniques ont permis de recenser 27 Asteraceae utilisées dans le traitement de diverses affections (Tableau 1). Ces Asteraceae médicinales sont regroupées en 20 genres et 7 tribus. Elles appartiennent à l'ordre des Asterales et au clade des Eudicotylédones. Elles appartiennent au sous-phyllum des Tracheophytinae et au phyllum des Embryophyta. Ce sont toutes des Spermaphytes, des Angiospermes représentées uniquement par des Dicotylédones. Le genre Vernonia, avec 6 espèces soit $22,24 \%$ des espèces recensées, constitue le genre le mieux représenté. Les genres Melanthera et Emilia avec chacun 2 espèces occupent la deuxième place. Les autres 17 genres, avec chacun une espèce respective, jouent également des rôles très importants. La tribu des Heliantheae et celle des Vernonieae avec chacune 9 espèces soit $33,33 \%$, constituent les tribus les mieux représentées. Les Eupatorieae avec 11,12\% viennent en deuxième position. Les Astereae et les Senecioneae avec chacune 2 espèces viennent en troisième position. Du point de vue des types morphologiques, les herbes sont majoritairement représentées avec $85,19 \%$. Les arbustes $(11,11 \%)$ et les lianes $(3,70 \%)$ sont également présents. Quant aux types biologiques, les Thérophytes avec 12 espèces sont les taxons les plus abondants. Ensuite viennent les Phanérophytes $(40,74 \%)$, les Hémicryptophytes $(11,11 \%)$ et une minorité de Chaméphytes (3,70\%). Parmi les Phanérophytes, on note une dominance des Nanophanérophytes par rapport aux Microphanérophytes. La répartition des espèces, par type phytogéographique, a montré que les espèces communes à la région Guinéo-Congolaise et à la région SoudanoZambienne (GC-SZ) sont les plus représentés avec 11 espèces soit 40,74\%. Elles sont suivies par les espèces de la région GuinéoCongolaise (GC), au nombre de 9 et celles de la région Soudano-Zambézienne (SZ), au nombre de 7. Du point de vue de leur statut, on dénombre 8 plantes sauvages et 19 plantes de milieux anthropiques comprennent 14 plantes rudérales, 4 plantes spontanées anthropophiles et 1 plante cultivée.

Les enquêtes ont porté sur 110 commerçants de plantes dont $93 \%$ sont de sexe féminin et $7 \%$ de sexe masculin. L'âge moyen est de 45 ans (16-75 ans). La majorité des herboristes, est analphabète $(65 \%)$ et le grand groupe ethnique Mandé avec 51 herboristes, est le plus représenté.

Les 27 Asteraceae recensées sont citées dans le traitement de 70 affections, symptômes, effets thérapeutiques, et phénomènes morbides, regroupés en 15 ensembles de maladie (paludisme, grossesse, maladies infantiles, maladies de la peau,....). Les Asteraceae médicinales les plus vendues et les plus citées par les commerçants dans le traitement des affections sont par ordre 
décroissant des fréquences de citation : Acanthospermum hispidum, Ageratum conyzoides et Vernonia cinerea, Bidens pilosa, Emilia sonchifolia, Mikania cordata, Tridax procumbens et Vernonia guineensis (Tableau 1). Les plantes recensées interviennent dans 57 recettes médicamenteuses dont $63,16 \%$ sont composées de recettes monospécifiques et $36,84 \%$ de recettes plurispécifiques (Tableau 2).

La valeur marchande des Asteraceae médicinales répertoriés varie de $365 \mathrm{FCFA} / \mathrm{kg}$ (0,61 USD) à 7810 FCFA/kg (12,96 USD) en fonction de la partie de la plante utilisée et de sa provenance.

\section{Caractéristiques des recettes médicamenteuses traditionnelles}

Les recettes médicamenteuses utilisées varient en fonction de l'importance relative des parties de plantes, du mode de préparation et d'administration (Tableau 2).

Les feuilles sont les parties majoritairement utilisées avec $43,18 \%$; ensuite viennent la plante entière, les rameaux, les racines et les écorces de tige avec respectivement $18,18 \%, 15,91 \%, 13,64 \%$ et $06,82 \%$. Les graines sont minoritairement utilisées avec $02,27 \%$. Pour certaines plantes comme Vernonia amygdalina et $V$. colorata diverses parties sont utilisées comme drogues (Feuilles, écorce de tige, racines, rameaux).

Le pétrissage, la décoction, l'expression et la macération sont les modes de préparation des remèdes traditionnels. Ainsi, la pâte, le décocté, le jus ou l'extrait et le macéré apparaissent comme les formes médicamenteuses. Le pétrissage est la technique de préparation la plus utilisée avec $38,60 \%$, ensuite vient la décoction avec $33,34 \%$. L'expression et la macération représentent chacune $14,03 \%$ des techniques de préparation.

La boisson est majoritairement utilisée dans 35,94\% des cas. Ensuite viennent le bain, le lavement et le pansement avec respectivement $15,63 \%, 10,94 \%$ et $09,38 \%$. La voie per os est la plus fréquemment utilisée dans $60,93 \%$ des cas contre $39,07 \%$ par la voie externe. Pour la voie per os, on note une plus grande utilisation de la voie orale dans $46,87 \%$ des cas contre $14,06 \%$ pour la voie anale et vaginale. Quant à la voie externe, le bain et le pansement demeurent les modes les plus utilisés.

\section{Bases scientifiques des utilisations thérapeutiques traditionnelles de 14 Asteraceae médicinales}

Des données phytochimiques issues de la littérature ont permis de comprendre le bien fondé des utilisations thérapeutiques traditionnelles de 10 Asteraceae médicinales que nous présentons dans le Tableau 3. Nous notons pour toutes les plantes la présence de l'un ou des composés chimiques tels que, les tanins, les alcaloïdes, les flavonoïdes, les terpènoïdes, les saponosides, les glycosides et les polyphénols. 


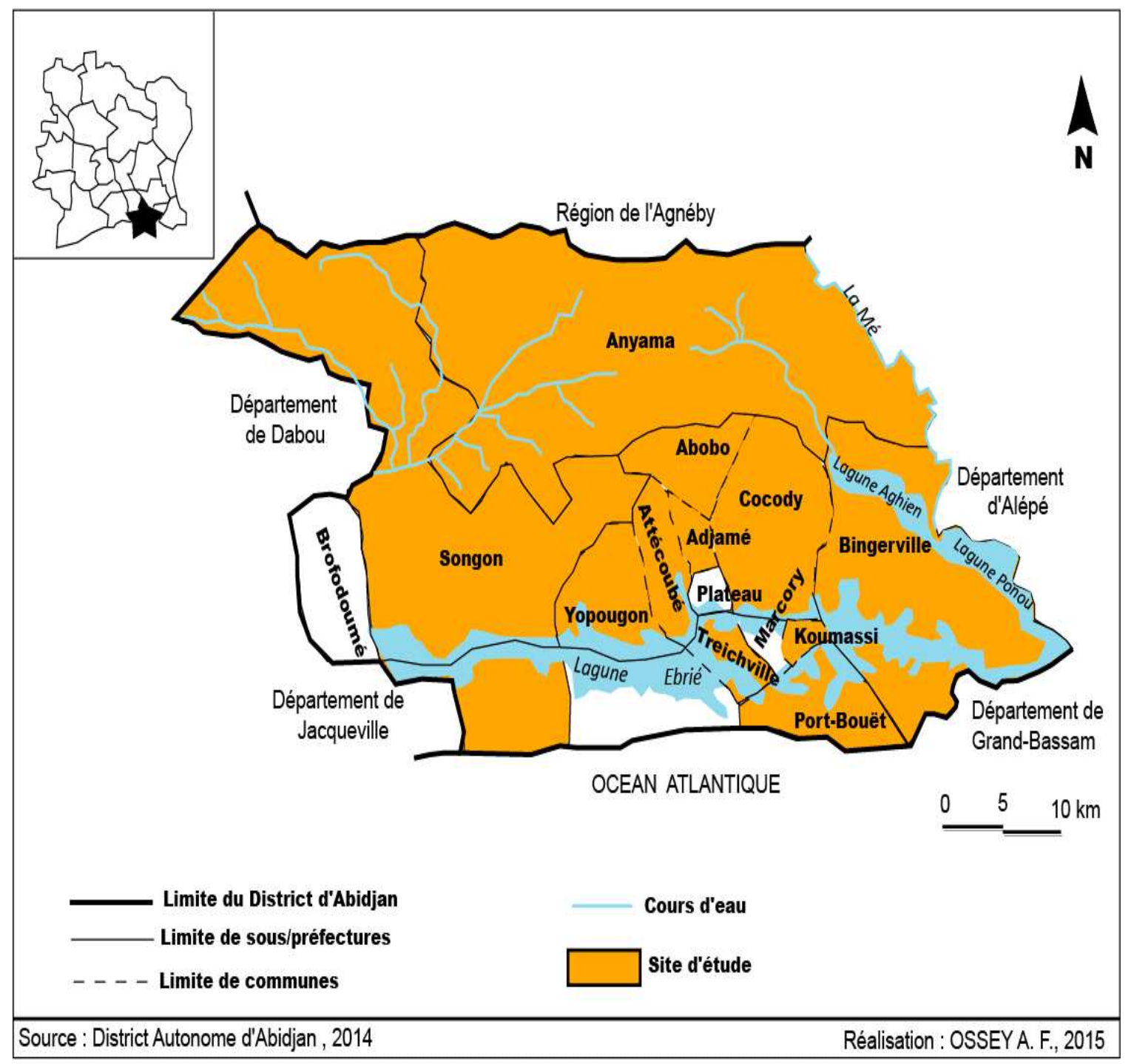

Figure 1 : Carte de localisation du district autonome d'Abidjan et des communes échantillonnées. 
Tableau 1 : Quelques noms vernaculaires et vertus thérapeutiques des Asteraceae médicinales répertoriées sur les marchés du district autonome d'Abidjan.

\begin{tabular}{|c|c|c|}
\hline Espèces végétales & Noms vernaculaires ivoiriens & Vertus thérapeutiques \\
\hline Acanthospermum hispidum DC. & Saraka-weini (Malinké) & $\begin{array}{l}\text { Paludisme, Ictère, Kyste ovarien, Perte blanche, Hypertension } \\
\text { artérielle, Maux de ventre, Parasite intestinaux, Grossesse, } \\
\text { Maladies infantiles }\end{array}$ \\
\hline Aedesia glaba (Klatt) O. Hoffm. & Konon-minan (Malinké) & Anémie, Fatigue générale, Maladie de la peau, Rhumatisme \\
\hline Ageratum conyzoides $\mathrm{L}$. & $\begin{array}{l}\text { Boclo/koun-gbéni/moussocôrôni-cougê } \\
\text { (Malinké); hoho (Abbey); pépein/n'métin-dou } \\
\text { (Akyé) }\end{array}$ & $\begin{array}{l}\text { Paludisme, Kyste ovarien, Perte blanche, Maux de ventre, Col de } \\
\text { l'utérus, Hémorroïde, Grossesse }\end{array}$ \\
\hline Aspilia africana (Pers.) C.D. Adams var. africana & Zeu-nanh (Akyé) ; Soumadibrou (Malinké) & Maux d'yeux, Toux, Maux de ventre, Maladies infantiles \\
\hline Bidens pilosa $\mathrm{L}$. & $\begin{array}{l}\text { Adzôpin-affifian/akyé-koi affifian (Akyé); } \\
\text { Alangoua (Baoulé); Chicôrô-magni (Abbey); } \\
\text { Noromi (Malinké) }\end{array}$ & $\begin{array}{l}\text { Panaris, Maladies infantiles, Toux, Plaies, Ulcères, Maladies de la } \\
\text { peau }\end{array}$ \\
\hline Centaurea praecox Oliv. et Hiern & Dondoutro (Malinké) & Diarrhée, Diabète \\
\hline Chromolaena odorata (L.) King et Robinson & Poinkêkê (Akyé) ; indépendant & Maux de ventre, Fatigue générale, Saignements, Ulcères, Plaies \\
\hline Chrysanthellum americanum (L.) Vatke & Aucun & Affections hépatiques, Maux d'oreilles, Hémorroïde \\
\hline Echinops longifolius A.Rich. & Aucun & Maux d'oreilles, Oreillons \\
\hline Eclipta prostrata (L.) L. & $\begin{array}{l}\text { N'da-loublé (Baoulé)； Missofi (Malinké); } \\
\text { Gnikiè (Akyé) }\end{array}$ & Asthme, Paludisme, Diabète, Plaies, Maladies infantiles \\
\hline Emilia praetermissa Milne-Readhead & Tchocoli-baté (Akyé) & Maux de ventre, Paludisme, Maladies infantiles, Grossesse \\
\hline Emilia sonchifolia (L.) DC. & Tchocoli-baté (Akyé) ; Gnon-vrai (Malinké) & $\begin{array}{l}\text { Maux de ventre, Parasites intestinaux, Maladies infantiles, } \\
\text { Grossesse }\end{array}$ \\
\hline Erigeron floribundus (H.B. et K.) Sch. Bip. & $\begin{array}{l}\text { Manôgô-dachi ou Banougou (Malinké) ; } \\
\text { Ayôrhô (Abbey) }\end{array}$ & Maladies de la peau, Kyste ovarien, Perte blanche \\
\hline Ethulia conyzoides L. & Chôkô (Malinké) & Maladies infantiles, Fatigue générale, Anémie, Toux \\
\hline Melanthera elliptica O. Hoffm. & Aucun & Toux, Paludisme, Maux de ventre \\
\hline Melanhera scandens (Schum. et Thonn.) Roberty & Zogno (Bété) & Toux, Paludisme, Fièvre \\
\hline
\end{tabular}


A. B. YAPI et al. / Int. J. Biol. Chem. Sci. 9(6): 2633-2647, 2015

Microglossa pyrifolia (Lam.) O. Ktze

Mikania cordata var. chevalieri C.D. Adams

Struchium sparganophora (L.) O. Ktze

Synedrella nodiflora Gaertn.

Tridax procumbens $\mathrm{L}$.

Vernonia ambigua Kotschy et Peyr.

Vernonia amygdalina Del.

Vernonia cinerea (L.) Less.

Vernonia colorata (Wild.) Drake

Vernonia guineensis Benth. var. guineensis

Vernonia perrottetii Sch. Bip.
Simoliè (Baoulé)

Appiè (Akyé); Kpitakpita-n'zalè (Baoulé); Paludisme, Ictère, Fièvre, Maladies de la peau, Maladies

Wouroutro (Malinké)

Bé-vê (Akyé) ; Kôm-missofi (Malinké) infantiles, Parasites intestinaux

Paludisme, Sinusite, Grossesse, Kyste ovarien

Koudjè-souramousso/djomo-djomo (Malinké)； Fatigue générale, Grossesse, Avortement, Insuffisance rénale

Assadjobo (Baoulé)

Aucun

Aucun

Abovi ou Abowi ou Abowoué ou Aovi Paludisme, Ictère, Hypertension artérielle, Maladies de la peau,

(Baoulé) ; So-kôssafounan (Malinké); Tôzô Fatigue générale

(Akyé) ; Viarô-vie (Abbey)

Gon-wonwon (Malinké)

Toux, Plaies, Maladies de la peau, Rhume, Fièvre, Hépatite virale, Anti-poison (vénin)

Blo-abovi ou Blo-abowi ou Blo-abowoué ou Paludisme, Ictère, Hypertension artérielle, Fatigue générale, Blo-aovi (Baoulé) ; Kongo-kôssafounan Fièvre jaune

(Malinké) ; Tôzô (Akyé)

Darama-kou ou Garabakou (Malinké), Koya Paludisme, Hypertension artérielle, Diabète, Grossesse, Maladies (Baoulé)

infantiles, Fièvre typhoïde

Paludisme, Hypertension artérielle, Affections hépatiques

Tableau 2 : Techniques de préparation, voies et modes d'administration et parties des Asteraceae médicinales utilisées comme drogue.

\begin{tabular}{|c|c|c|c|c|}
\hline Espèces & Parties de la plante utilisées & Techniques de préparation & Voies d'administration & Modes d'administration \\
\hline${ }^{* 2}$ Acanthospermum hispidum & Plante entière & Décoction/Pétrissage & Per os/Voie externe & Boisson/Bain/Friction \\
\hline Aedesia glaba & Feuilles & Décoction & Per os/Voie externe & Boisson/Bain/Friction \\
\hline${ }^{* 1}$ Ageratum conyzoides & Feuilles & Décoction/Pétrissage/Expression & Per os/Voie externe & Boisson/Bain/Instillation nasale \\
\hline Aspilia africana & Feuilles & $\begin{array}{c}\text { Décoction/Pétrissage/Expression } \\
2640\end{array}$ & Per os/Voie externe & Boisson/Bain/Instillation oculaire \\
\hline
\end{tabular}


A. B. YAPI et al. / Int. J. Biol. Chem. Sci. 9(6): 2633-2647, 2015

\begin{tabular}{|c|c|c|c|c|}
\hline Bidens pilosa & Feuilles & Pétrissage/Expression & Per os/Voie externe & Boisson/Friction/Pansement \\
\hline${ }^{* 1}$ Centaurea praecox & Feuilles/Racines & Décoction & Per os & Boisson \\
\hline Chromolaena odorata & Feuilles & Décoction/Pétrissage/Expression & Per os/Voie externe & Boisson/Friction/Pansement \\
\hline Chrysanthellum americanum & Plante entière & Décoction/Pétrissage & Per os & Boisson \\
\hline Echinops longifolius & Rameaux/Graines & Décoction/Pétrissage & Per os & Instillation auriculaire \\
\hline Eclipta prostrata & Feuilles & Décoction/Pétrissage & Per os/Voie externe & Boisson/Pansement \\
\hline Emilia praetermissa & Feuilles & Décoction/Pétrissage & Per os & Boisson \\
\hline Emilia sonchifolia & Plante entière & Décoction/Pétrissage & Per os/Voie externe & Boisson/Pansement \\
\hline Erigeron floribundus & Feuilles & Macération/Décoction & Voie externe & Bain/Toilette intime \\
\hline Ethulia conyzoides & Feuilles & Décoction/Pétrissage/Expression & Per os/Voie externe & Boisson/Aspersion/Badigeonnage \\
\hline Melanthera elliptica & Feuilles & Pétrissage/Expression & Per os & Boisson/Instillation nasale \\
\hline Melanhera scandens & Feuilles & Pétrissage/Expression & Per os & Boisson/Instillation nasale \\
\hline Microglossa pyrifolia & Feuilles/Racines & Décoction/Pétrissage & Voie externe & Boisson/Bain/Lavement \\
\hline Mikania cordata & Plante entière & Décoction & Voie externe & Boisson/Bain \\
\hline Struchium sparganophora & Feuilles & Pétrissage/Expression & Per os/Voie externe & Instillation nasale/Aspersion/Lavement \\
\hline${ }^{* 1}$ Synedrella nodiflora & Feuilles & Pétrissage & Per os & Lavement \\
\hline${ }^{* 1}$ Tridax procumbens & Plante entière & Macération/Pétrissage & Per os/Voie externe & Boisson/Instillation oculaire/Pansement \\
\hline Vernonia ambigua & Feuilles/Rameaux & Décoction/Pétrissage & Per os/Voie externe & Bain/Lavement \\
\hline Vernonia amygdalina & $\begin{array}{l}\text { Feuilles/Ecorce } \\
\text { tige/Racine/Rameaux }\end{array}$ & Macération/Décoction/Pétrissage & Per os/Voie externe & Boisson/Bain/Lavement/Badigeonnage \\
\hline${ }^{* 2}$ Vernonia cinerea & Plante entière & Macération/Pétrissage & Per os/Voie externe & Boisson/Bain/Pansement \\
\hline${ }^{2}$ Vernonia colorata & $\begin{array}{l}\text { Feuilles/Ecorce } \\
\text { tige/Racines/Rameaux }\end{array}$ & Macération/Décoction/Pétrissage & Per os/Voie externe & Boisson/Bain/Lavement/Badigeonnage \\
\hline Vernonia guineensis & Feuilles & Macération & Per os & Boisson \\
\hline Vernonia perrottetii & $\begin{array}{l}\text { Rameaux/Ecorce de } \\
\text { tige/Racines }\end{array}$ & Décoction/Pétrissage & Per os & Boisson/Lavement \\
\hline
\end{tabular}

*1 : Recette bispécifique faisant intervenir: Capsicum frutesc

(Asclepiadaceae) ou Sesamum alatum Thonning (Pedaliaceae).

$*^{2}$ : Recette plurispécifique faisant intervenir : Momordica charantia L. (Cucurbitaceae), Phyllanthus amarus Schumach. \& Thonn. (Euphorbiaceae), Pupalia lappacea (L.) Juss. (Amaranthaceae), Heteropogon contortus (L.) P. Beauv. ex Roem. \& Schult. (Poaceae). 
A. B. YAPI et al. / Int. J. Biol. Chem. Sci. 9(6): 2633-2647, 2015

Tableau 3: Tri phytochimique de 10 Asteraceae médicinales issues de la littérature.

\begin{tabular}{|c|c|c|c|c|c|c|c|c|c|}
\hline \multirow{3}{*}{ Espèces } & \multirow{3}{*}{ Auteurs } & \multicolumn{8}{|c|}{ Groupes chimiques } \\
\hline & & \multirow[t]{2}{*}{ Glycosides } & \multirow[t]{2}{*}{ Saponosides } & \multirow[t]{2}{*}{ Flavonoïdes } & \multirow{2}{*}{$\begin{array}{l}\text { Terpènes/ } \\
\text { Stérols }\end{array}$} & \multirow[t]{2}{*}{ Polyphénols } & \multirow[t]{2}{*}{ Alcaloïdes } & \multicolumn{2}{|c|}{ Tanins } \\
\hline & & & & & & & & Cat & $\mathbf{G a}$ \\
\hline Ac & Anup et al., 2012 & + & + & + & + & - & + & + & + \\
\hline $\mathrm{Ag}$ & N'Guessan, 2008 & - & + & + & + & + & + & - & - \\
\hline $\mathrm{Co}$ & & - & + & + & + & + & + & + & - \\
\hline $\mathrm{Bd}$ & Bilanda et al., 2004 & - & - & - & - & + & + & - & - \\
\hline Es & Nwadinigwe, 2009 & + & + & + & - & - & - & - & - \\
\hline $\mathrm{Sn}$ & Bhogaonka et al., 2011 & - & + & + & + & - & + & - & - \\
\hline $\mathrm{Tp}$ & Sneha et Ruchi, 2010 & - & + & + & - & - & + & + & + \\
\hline $\mathrm{Va}$ & Ebenezer et Olatunde, 2011 & - & + & + & + & - & + & - & - \\
\hline Vci & Ahsanul et al., 2012 & + & - & - & + & - & - & - & - \\
\hline Vco & Mbodj, 2003 & - & + & - & + & - & + & + & + \\
\hline
\end{tabular}

Cat : Catéchiques, Gal : Galliques ; + : Présence, - : Absence

Ac : Acanthospermum hispidum, Ag : Ageratum conyzoides, $\mathbf{C o}:$ Chromolaena odorata, Bd : Bidens pilosa, Es : Emilia sonchifolia, $\mathbf{S n}:$ Synedrella nodiflora, Tp : Tridax procumbens, Va : Vernonia amygdalina, Vci : Vernonia cinerea, Vco : Vernonia colorata. 


\section{DISCUSSION}

Ces travaux consistaient à recenser et collecter les informations sur les plantes médicinales de la famille botanique des Asteraceae et commercialisées sur les marchés $\mathrm{du}$ district autonome d'Abidjan. Elle est réalisée auprès de 110 commerçants de plantes à travers une série d'enquêtes ethnobotaniques. Les résultats de cette étude ont montré que la commercialisation des plantes médicinales dans les marchés d'Abidjan occupe les hommes et les femmes dont l'âge varie de 16 à 75 ans. Les commerçantes sont majoritairement représentées (93\%). Cela s'explique par le fait qu'en Côte d'Ivoire, la vente d'articles au marché est réservée habituellement aux femmes. Les commerçants âgés de plus de 36 ans sont les plus nombreux parce qu'ils ont plus de connaissances sur les vertus des plantes médicinales. Les herboristes disent avoir acquis l'art médical traditionnel par apprentissage auprès de leur père ou de leur mère. C'est donc une transmission du savoir médical traditionnel de génération en génération. Cette observation a été aussi faite par N'Guessan et al. (2011). Parmi les espèces recensées, les thérophytes sont les plus nombreux. Ces résultats reflètent les types biologiques d'Asteraceae recensées en Côte d'Ivoire dont 37,2\% de thérophytes (AkéAssi, 2001). Cette étude a montré que les herbes sont massivement utilisées. Cela serait dû au fait que ces plantes se rencontrent fréquemment, dans l'environnement immédiat des utilisateurs et par la facilité d'accès aux différents organes. Ce résultat concorde avec celui obtenu par Aké-Assi (2011) qui a montré que les herbes représentent $75 \%$ des plantes médicinales traditionnellement employées dans la couverture des soins de santé primaire, en Afrique. Cette étude a aussi montré que les espèces communes à la région GC-SZ sont les plus importantes contrastant avec ceux d'Adomou et al. (2012) qui ont montré une forte représentativité des espèces Pantropicales au sud du Bénin dans le marché
d'Abomey-Calavi. Les plantes de milieux anthropiques $(70 \%)$ sont majoritairement retrouvées et constituent la grande partie des Asteraceae médicinales répertoriées. Cela témoigne de l'état de dégradation avancée du patrimoine forestier originel. Les Asteraceae médicinales répertoriées sur les marchés, sont citées dans le traitement de diverses maladies dont la plus couramment citée est le paludisme. Ce résultat pourrait s'expliquer par le fait que le paludisme est une parasitose qui sévit dans les régions tropicales, en particulier en Afrique subsaharienne. Cette maladie est considérée, aujourd'hui, comme une cause de pauvreté (OMS, 2004). En Côte d'Ivoire, selon le PNLP (2009) le paludisme constitue la première cause de morbidité, d'hospitalisation et de mortalité dans les services régionaux de pédiatrie $(33 \%$ des décès)

L'analyse de la fréquence de citation des plantes a montré que Acanthospermum hispidum, Ageratum conyzoides Vernonia cinerea, Bidens pilosa, Emilia sonchifolia, Mikania cordata, Tridax procumbens, Vernonia guineensis, Euphorbia hirta, sont les plus fréquemment citées dans le traitement des affections et les mieux répertoriées sur les marchés du district d'Abidjan. Par ailleurs, ces plantes seraient sans doute parmi les plus efficaces. Selon Fleischer et al. (2003), Acanthospermum hispidum est très utilisée en médecine populaire au Brésil. Elle est citée dans le traitement de plusieurs maladies (asthme, bronchite, migraine,...). Selon ces mêmes auteurs, Ageratum conyzoides est utilisée dans le traitement des hyperthermies, des hémorragies du post-partum et ceci en association avec Parquetina nigrescens. Selon N'Guessan (2008), Ageratum conyzoides est utilisée dans le traitement des taches dermiques, de la métrorragie, du paludisme, des maux d'yeux... Les convergences de ses résultats avec ceux d'autres auteurs ne justifient pas nécessairement l'efficacité des recettes, mais représentent un élément de fiabilité pour nos données. Elles peuvent 
néanmoins orienter les phytochimistes et pharmacologues dans le choix des plantes pour appuyer les usages traditionnels. Cette étude a révélé que les feuilles sont les organes les plus utilisés comme drogue pour les préparations médicamenteuses. Selon certaines études (Ouattara, 2006 ; N'Guessan, 2008), les feuilles étaient les organes les plus sollicitées comme drogues pour la préparation des recettes médicamenteuses traditionnelles. Selon Betti (2001), le prélèvement de $50 \%$ des feuilles d'un arbre n'affecte pas de façon significative, la survie de celui-ci. Par contre, selon le même auteur, l'écorçage est stressant tout comme l'ébranchage et l'abattage qui s'avèrent nocifs pour la plante, sont à l'origine de la menace de disparition des espèces. De plus, les feuilles sont connues comme le lieu de synthèse des métabolites secondaires du végétal (Mangambu et al., 2014). Le pétrissage est la technique de préparation des recettes médicamenteuses la plus employée. Ce résultat est conforme à celui obtenu par N'Guessan (2008). Cela pourrait s'expliquer par le fait que la pâte issue du pétrissage est rapide à obtenir et facile à conserver par séchage. Les préparations sont majoritairement prescrites en boisson (voie orale). Des travaux (Ouattara, 2006 ; N'Guessan, 2008) ont montré que la boisson est le mode d'administration le plus sollicité en médecine traditionnelle. Pour des maladies liées à des infections bactériennes, fongiques et/ou parasitaires localisées dans des organes profonds, le transit des médicaments par l'appareil digestif facilite leur assimilation (Tra Bi et al., 2008) et leur action. Cette étude a également révélé que la plus faible valeur marchande des Asteraceae médicinales est plus élevée par rapport au prix d'achat de l'huile de palme (325 FCFA) et du caoutchouc (320 FCFA). La plus forte valeur marchande qui est de $7810 \mathrm{FCFA} / \mathrm{kg}$ équivaut à 7,81 fois celui du cacao ivoirien acheté bord-champ (1,66 USD), en octobre 2015. La valeur marchande de ces plantes est nettement supérieure à celle des principales cultures d'exportation qui ont une part importante dans l'économie nationale. De ce fait, la vente de plantes médicinales peut être considérée comme une activité lucrative.

Il a été procédé à une validation des plantes recensées, en recherchant, pour quelques-unes, la composition phytochimique qui permettraient d'expliquer leurs effets pharmacologiques. Les tests phytochimiques réalisés sur quelques espèces issues de la littérature indiquent la présence de tanins, de flavonoïdes, de polyphénols, d'alcaloïdes de polyterpenes de glycosides et de saponines. Il a été rapporté dans la littérature que les alcaloïdes, (N'Guessan, 2008), seraient responsables des propriétés antipaludiques de certaines plantes comme Ageratum conyzoides, Bidens pilosa. Selon Mbodj (2003), les études chimiques effectuées sur Vernonia colorata, ont montré la présence d'alcaloïdes, de tanins, d'hétérosides cardiotoniques, de saponosides. Selon le même auteur, la présence de ces composés chimiques, seraient à l'origine des propriétés pharmacologiques qui sont attribuées à cette plante. Les composés chimiques contenus dans les plantes, pourraient justifier leur utilisation empirique dans diverses médecines traditionnelles.

\section{Conclusion}

Cette étude a permis de faire une évaluation de la diversité des Asteraceae médicinales commercialisées sur les marchés du district autonome d'Abidjan et utilisées dans le traitement traditionnel de plus de diverses affections. Parmi les espèces répertoriées, on note une prédominance de plantes de milieux anthropisés. Les feuilles sont majoritairement les organes utilisés dans la préparation des recettes médicamenteuses. Les modes de préparation sont multiples, mais le pétrissage est le plus courant. L'administration des médicaments traditionnels proposés se fait surtout par voie orale. De plus, l'étude a mis en évidence la valeur marchande des Asteraceae médicinales. 
Les effets curatifs induits par les plantes étudiées, sont le fait de divers groupes chimiques (alcaloïdes, flavonoïdes, glycosides...) qui constituent la base scientifique de leur utilisation traditionnelle. Cette étude est réalisée dans la perspective de rechercher les principes actifs des indications thérapeutiques reçues à travers des essais biologiques et chimiques. L'usage des méthodes plus perfectionnées du screening phytochimique et l'évaluation de l'activité pharmacologique à travers des tests antimicrobiens des différents extraits seraient importants pour compléter cette étude ethnobotanique.

\section{CONFLITS D'INTERETS}

Les auteurs déclarent qu'ils n'ont aucun conflit d'intérêts.

\section{CONTRIBUTIONS DES AUTEURS}

YAB a réalisé les enquêtes ethnobotaniques et a contribué à la rédaction du manuscrit ; NJK a contribué à la réalisation de la fiche d'enquête et à la rédaction du manuscrit ; BYFN a contribué à l'analyse des différentes pathologies traitées par ses plantes; GNZ a contribué par sa lecture à l'amélioration et à la validation du manuscrit

\section{REMERCIEMENTS}

Les auteurs remercient l'ensemble des herboristes des marchés visités et les mairies $\mathrm{du}$ district autonome d'Abidjan pour leur accessibilité, leur disponibilité et leur franche collaboration à cette étude. Ils remercient, également, Monsieur ASSI Yapo Jean, Technicien botaniste au C.N.F, de 1'Université Félix HOUPHOUET-BOIGNY de Cocody pour sa contribution à l'étude de reconnaissance des Asteraceae et à l'identification des plantes recensées.

\section{REFERENCES}

Fleischer TC, Ameade EP, Sawer IK. 2003. Antimicrobial activity of the leaves and flowering tops of Acanthospermum hispidum. Fitoterapia, 74: 130-132.

Adomou AC, Yedomonhan H, Djossa B, Legba SI, Oumorou M, Akoegninou A. 2012. Etude ethnobotanique des plantes médicinales vendues dans le marché d'Abomey Calavi au Bénin. Int. J. Biol. Chem. Sci., 6(2): 745-772. DOI : http://dx.doi.org/10.4314/ijbcs. v6i2.18

Ahsanul H, Musfizur H, Atanu D, Bilkis B, Yousuf A, Helal M. 2012. Phytochemical investigation of Vernonia cinerea (Astéraceae). Journal of Applied Pharmaceutical Science, 2(6): 79-83. DOI : 10.7324/JAPS.2012.2617

Ake-Assi L. 2001. Flore de la Côte-Ivoire : catalogue, systématique, biogéographie, et écologie. Boissiera 57.

Ake-Assi L. 2011. Abrégé de médecine et pharmacopées africaines: quelques plantes employées traditionnellement dans la couverture des soins de santé primaire. Edition NEI/CEDA (Côte d'Ivoire), $157 \mathrm{p}$.

Anup KC, Amit VG, Karuna BS. 2012. Phytopharmacological review on Acanthospermum hispidum. Journal of Applied Pharmaceutical Science, 2(1): 144-148.

Azokou A. 2010. Activité Biolarvicide de quelques espèces végétales de côte d'Ivoire contre les moustiques Culex quiquefasciatus et Anopheles gambiae, vecteurs de maladies. DEA de G.V.R.N., UFR-SN. Université Abobo-Adjamé (Abidjan-Côte d'Ivoire), p 73.

Bhogaonka PY, Dagawal DS, Gvish A, Radhabai S, Anjangaon S. 2011. Pharmacognostic studies and antimicrobial activity of Synedrella nodiflora (L.) Gaertn. Bioscience Discovery, 2(3): 317-321.

Betti JL. 2001. Usages traditionnels et vulnérabilité des plantes médicinales dans la réserve de biosphère du Dja, Cameroun. Thèse de Doctorat, Université Libre de Bruxelles, Belgique, p. 87. 
Bilanda DC, Dimot T, Mbacham FW, Evehe MS, Muluh J, Njufutie N. 2004. Effets in vitro de Bidens pilosa sur la chloroquinorésistance de Plasmodium falciparum. Pharm. Méd. Trad. Afr., XI ${ }^{\text {ème }}$ colloque du CAMES, Yaoudé (Cameroun), 13 : 21-28.

Ebenezer OF, Olatunde O. 2011. Antioxidative and chemopreventive properties of Vernonia amygdalina and Garcinia biflavonoid. International Journal of Environmental Research and Public Health, 8(6): 2533-2555. DOI: 10.3390/ijerph8062533.

Eddouks M, Ouahidi ML, Farid O, Moufid A, Khalidi A, Lemhadri A. 2007. L'utilisation des plantes médicinales dans le traitement du diabète au Maroc. Phytothérapie, 5: 194-203. DOI: 10.1007/s 10298-007-0252-4.

Gning SB, Thiam M, Fall F, Ba-Fall K, Mbaye PS, Foucarde L. 2007. Le diabète sucré en Afrique subsaharienne: aspects épidémiologiques, difficultés de prise en charge. Médecine Tropicale, 67: 607-611.

Gueye M, Cisse A, Diatta CD, Diop S, Koma S. 2012. Etude ethnobotanique des plantes utilisées contre la constipation chez les Malinké de la communauté rurale de Tomboronkoto, Kédougou (Sénégal). Int. J. Biol. Chem. Sci., 6(2): 778-779.

DOI : http://dx.doi.org/10.4314/ijbcs.v6i2.19

Hossain P, Kawar B, Nahas M. 2007. Obesity and diabetes in developing world. A growing challenge. N. England Journal of Medicine, 356: 213-215. DOI: 10.1056/NEJMp068177.

Klotoé JR, Dougnon TV, Koudouvo K, Atègbo J-M, Loko $\mathrm{F}$, Akoègninou $\mathrm{A}$, Aklikokou K, Dramane K, Gbeassor M. 2013. Ethnopharmacological survey on antihemorrhagic medicinal plants in South of Benin. European Journal of Medicinal Plants, 3(1): 40-51.

Lebri M, Bahi C, Fofié NBY, Gnahoue G, Lagou SM, Achibat H, Yapi A, Zirihi
GN, Coulibaly A, Hafid A, Khouili M. 2015. Analyse phytochimique et évaluation de la toxicité aiguë par voie orale chez des rats de l'extrait total aqueux des feuilles de Abrus precatorius Linn (Fabaceae). Int. J. Biol. Chem. Sci., 9(3): 1470-1476. DOI : http://dx.doi.org/10.4314/ijbcs.v9i3.29.

Mangambu M, Mushagalusa K, Kadima N. 2014. Contribution à l'étude phytochimique de quelques plantes médicinales antidiabétiques de la ville de Bukavu et ses environs (Sud-Kivu, R.D.Congo). Journal of Applied Biosciences, 75: 6211-6220. DOI : http://dx.doi.org/10.4314/jab.v75i1.7

Mbodj NA. 2003. Etude de l'activité antidiabétique des extraits acétoniques, méthanoliques, et hexaniques de Vernonia colorata (Willd.) Drake composées chez les rats wistars. Thèse pour obtenir le grade de docteur en pharmacie. Université Cheikh Anta Diop de Dakar. Faculté de médecine, pharmacie et d'odonto-stomatologie. Département de pharmacie, p 61.

N'Guessan K. 2008. Plantes médicinales et pratiques médicales traditionnelles chez les peuples Abbey et Krobou du Département d'Agboville (Côte d'Ivoire). Thèse de Doctorat d'Etat, Université de Cocody-Abidjan, U.F.R. Biosciences, Laboratoire de Botanique, p 235.

N'Guessan K, Soro D, Amon A. 2011. Plantes utilisées en médecine traditionnelle dans le traitement des maladies cardiovasculaires, en pays Abbey et Krobou dans le sud de la Côte d'Ivoire. Phytothérapie, 9: 199-208. DOI : 10.1007/s10298.011-0636-3.

Nwadinigwe AO. 2009. Antimicrobial activities of some fractions of the extract of Emilia sonchifolia (L.) DC. (Asteraceae). Plant Products Research Journal, 13: 1119-2283. DOI : http://dx.doi.org/10.4314/pprj.v13i1.6581 3 
OMS. 2004. Le paludisme: une des principales causes de décès et de pauvreté des enfants en Afrique. http://rbm.who.int/docs/rps_publications/ unicef_malaria_fr.pdf.

Ouattara D. 2006. Contribution à l'inventaire des plantes médicinales significatives utilisées dans la région de Divo (sud forestier de la Côte-d'Ivoire) et à la diagnose du poivrier de Guinée : Xylopia aethiopica (Dunal) A Rich (Annonaceae). Thèse de Doctorat de l'Université de Cocody, Abidjan (Côte, d'Ivoire), UFR Biosciences, p 184.

PNLP. 2009. Rapport annuel sur la situation du paludisme. Programme National de Lutte contre le Paludisme, Ministère de la Santé et de l'Hygiène Publique. République de Côte d'Ivoire, 163 p.
Sangaré MM, Sina H, Dougnon J, Bayala B, Ategbo J-M, Dramane KL. 2012. Etude ethnobotanique des plantes hépatotropes et de l'usage traditionnel de Gomphrena celosioides Mart. (Amaranthaceae) au Bénin. Int. J. Biol. Chem. Sci., 6(6): 5008-5021.

DOI http://dx.doi.org/10.4314/ijbcs.v6i6.20

Sneha M, Ruchi S. 2010. Pharmacology of Tridax procumbens a Weed Review. International Journal of Research Pharm. Tech., 2(2): 1391-1394.

Tra Bi FH, Irié GM, Kohué CC, N'Gaman, Clejesson HB, Mohou. 2008. Etudes de quelques plantes thérapeutiques utilisées dans le traitement de l'hypertension artérielle et du diabète: deux maladies émergentes en Côte d'Ivoire. Sciences \& Nature, 5(1):39-48. 